\title{
Esquemas maladaptativos tempranos en pacientes hipertensos controlados en Segovia, Colombia
}

\author{
Ana Yajaira Restrepo ${ }^{\star}$, Renato Zambrano Cruz ${ }^{\star \star}$
}

* Psicóloga. Coordinadora local Segovia-Remedios de la Agencia Nacional para la Superación de la Pobreza Extrema.

Correo electrónico: anayajis88@hotmail.com

** Magíster en Lingüística. Docente del Departamento de Psicología, Universidad de Antioquia. Correo electrónico: renatozambrano@gmail.com

Recibido: 1 de octubre del 2013

Aceptado: 30 de octubre del 2013

Cómo citar este artículo: Restrepo, $A$. Y. y Zambrano Cruz, R. (2013). Esquemas maladaptativos tempranos en pacientes hipertensos controlados en Segovia, Colombia. Pensando Psicología, 9(16), 69-75.

\begin{abstract}
Resumen
Este estudio identifica la presencia de esquemas maladaptativos tempranos (ЕMT) en pacientes hipertensos controlados de la ESE Hospital San Juan de Dios del municipio de Segovia (Colombia). Este trabajo se realiza a partir de la perspectiva teórica de Jeffrey Young, quien sostiene que los EMT sirven como filtros a través de los cuales se procesa la información de la vida diaria, lo cual repercute en la manera de afrontar cada situación. Con el tipo de investigación transversal no experimental, se describe y se establece comparación de los EMT mediante el Cuestionario de Esquemas de Young YSQ - L2 (Castrillón, Chaves, Ferrer, Londoño, Maestre, Marín y Schnitter, 2005) entre dos grupos en los cuales participaron 73 personas con diagnóstico de hipertensión y 64 personas normotensas con características sociodemográficas similares. Los resultados muestran como esquemas maladaptativos tempranos prevalecientes: abandono, vulnerabilidad al daño y a la enfermedad e inhibición emocional. Al compararlos con el segundo grupo, la vulnerabilidad al daño y a la enfermedad fue el único esquema que obtuvo puntuaciones mayores.
\end{abstract}

Palabras clave: esquemas, esquemas maladaptativos tempranos, hipertensión arterial, psicología de la salud.

\section{Early maladaptive systems in controlled hypertensive patients in Segovia, Colombia}

\begin{abstract}
This study identifies the presence of early maladaptive schemas in controlled hypertensive patients at the Hospital San Juan de Dios in the municipality of Segovia (Colombia). The work was carried out based on the theoretical perspective of Jeffrey Young, who argues that Ems serve as filters through which information is processed in daily life, and which affect how each situation is dealt with. Using a nonexperimental cross-sectional research study, EMs are described and compared through use of the Young Questionnaire YsQ - L2 (Castrillón, Chaves, Ferrer, Londoño, Master, Marin y Schnitter, 2005) applied to two groups, consisting of 73 people diagnosed with hypertension and 64 normotensive subjects with similar demographic characteristics. The results show prevalent early maladaptive schemas involving abandonment, vulnerability to injury and illness and emotional inhibition. When compared with the second group, vulnerability to injury and disease was the only area that obtained higher scores.
\end{abstract}

Keywords: schemas, early maladaptive schemas, arterial hypertension, health psychology.

Esquemas mal-adaptativos precoces em pacientes hipertensos controlados em Segovia, Colômbia

\section{Resumo}

Este estudo identifica a presença de esquemas mal-adaptativos precoces em pacientes hipertensos controlados da ESE Hospital San Juan de Dios do município de Segovia (Colômbia). Este trabalho se realiza a partir da perspectiva teórica de Jeffrey Young, que sustenta que os EMTs servem como filtros pelos quais se processa a informação da vida diária, o qual repercute na maneira de afrontar cada situação. Com o tipo de pesquisa transversal não experimental, descreve-se e estabelece-se comparação dos EMTs mediante o Questionário de Esquemas de Young YsQ - L2 (Castrillón, Chaves, Ferrer, Londoño, Maestre, Marín e Schnitter, 2005) entre dois grupos dos quais participaram 73 pessoas com diagnóstico de hipertensão e 64 pessoas normotensas com características sociodemográficas similares. Os resultados mostram como EMTs prevalecentes: abandono, vulnerabilidade ao dano e à doença e inibição emocional. Ao compará-los com o segundo grupo, vulnerabilidade ao dano e à doença, foi o único esquema que obteve pontuações maiores.

Palavras-chave: esquemas, esquemas mal-adaptativos precoces, hipertensão arterial, psicologia da saúde. 
La Presión Arterial (PA) es la fuerza o tensión que la sangre ejerce sobre las paredes de las arterias al pasar por ellas. Esta presión alcanza su valor máximo durante la sístole ventricular (presión sistólica) y el más bajo durante la relajación cardíaca (presión diastólica). Aunque la presión arterial de un individuo varía con las actividades de la vida diaria, los valores promedio o "normales" generalmente están cerca de $140 \mathrm{mmHg} / 80 \mathrm{mmHg}$ (Aristizábal et al., 2006). En un individuo, los factores genéticos, ambientales y demográficos contribuyen a la variación de la PA. Tradicionalmente se ha aceptado que entre un $90-95 \%$ de los humanos hipertensos presentan una forma de hipertensión (HTA) en la que no es posible identificar una causa orgánica que explique la elevación de la PA (Aristizábal, García, McEwen, Caulfield, Méndez, Medina, Zapata y Correa, 2006); dicho tipo de HTA es denominada esencial o primaria (Miguel-Tobal, Cano-Vindel, Casado Morales y Escalona Martínez, 1994). Cuando se habla de hipertensión estamos refiriéndonos a una patología que pertenece al grupo de enfermedades coronarias sobre las que existe mayor implicación de factores psicosociales (Miguel-Tobal et al., 1994).

Desde la psicología psicosomática y de la salud, se ha hablado de la presencia de factores psicosociales influyentes en diferentes patologías, incluidas las de orden coronario: la hipertensión, la taquicardia, la enfermedad de Raynaud, la úlcera, el síndrome de intestino irritable, la colitis ulcerosa, el asma, la dermatitis, las cefaleas, etcétera (Miguel-Tobal et al., 1994). De hecho, algunos autores como Meyer Friedman y Ray Rosenman (1959, citados en Miguel-Tobal et al., 1994), crearon un perfil con las características psicológicas de acuerdo con una personalidad en común encontrada en individuos que sufrían de alguna enfermedad cardiaca, de la cual los comportamientos más comunes fueron la competitividad, el perfeccionismo, la ambición, la urgencia temporal, una tendencia a sobrecargarse de actividades y desempeñar cargos de responsabilidad, impaciencia, frustración frente a la realidad, reflejados con hostilidad y agresividad.

Teniendo en cuenta la implicación psicológica en la HTA y orientándonos desde una vertiente cognitiva, nos remitimos a los esquemas maladaptativos tempranos, definidos como una estructura extremadamente estable y duradera que se elabora durante la infancia, se desarrolla durante toda la vida del individuo y es disfuncional en alto grado. Los esquemas sirven como plantillas por medio de las cuales se procesará posteriormente la información. Serán, pues, elementos cognitivos que influirán de manera concreta en la forma en que los sujetos perciben el mundo y, como consecuencia, esta distorsión cogniti- va llevará al sujeto a padecer desadaptación o disfuncionalidad ante situaciones que no tendrían que ser así, ya que no se corresponden con la realidad (Young, 1999, citado en Castrillón et al., 2005).

Con base en lo anterior, el presente estudio evalúa la presencia de dichos esquemas maladaptativos tempranos en población hipertensa controlada de la ESE Hospital San Juan de Dios en el municipio de Segovia (Colombia), y se establece una comparación con un grupo de población normotensa con características similares (edad, estrato socioeconómico, estado civil, nivel académico).

\section{Método}

Esta investigación obedece a un diseño transversal enmarcándose en un enfoque cuantitativo con un alcance descriptivo-correlacional, puesto que además de describir los esquemas maladaptativos temprano (EMT) en la población estudiada, se establece también una relación entre la presencia de esquemas con el fenómeno de la hipertensión (Hernández Sampieri, Fernández Collao, y Baptista Lucio, 2003).

\section{Población y muestra}

Para este estudio se determinaron dos muestras; la primera está referida a la población hipertensa que asiste regularmente a los controles por enfermería a la ESE Hospital San Juan de Dios del municipio de Segovia, Colombia. De este primer grupo se determinó una muestra por oportunidad de 73 pacientes (Edad media: 56,2; Desviación estándar: 12,4).

El segundo grupo está constituido por 64 participantes normotensos; estos pacientes no debían padecer ninguna enfermedad coronaria, y habían de tener características similares al primer grupo (rango de edad, género, grado de escolaridad). Este segundo grupo fue seleccionado de personas que asisten al grupo del adulto mayor y al gimnasio municipal. La muestra total se conformó por un $82 \%$ de mujeres, así como un $42 \%$ de personas casadas seguidas por solteras (36\%), y el restante entre separadas, en unión libre y en estado de viudez. El $57,7 \%$ de las personas pertenecía al estrato 1 .

\section{Instrumento}

El cuestionario Young Schema Questionaire fue desarrollado por Jeffrey Young para evaluar los EMT. Posteriormente el mismo autor ha llevado a cabo revisiones 
teóricas de los esquemas y su forma de agrupación, dando lugar a diferentes versiones del cuestionario (Castrillón et al., 2005). Para establecer las propiedades psicométricas y la validez estructural del Cuestionario de esquemas de Young forma larga, se aplicó a 1.419 estudiantes universitarios de Medellín. El estudio encontró que la prueba tenía una estructura factorial que se organizaba en 11 factores y explicaban el 65\% de la varianza. Los factores encontrados fueron: abandono; insuficiente autocontrol/autodisciplina; desconfianza/ abuso; privación emocional; vulnerabilidad al daño y a la enfermedad; autosacrificio; estándares inflexibles (I y II, factores séptimo y octavo); inhibición emocional; derecho y entrampamiento. Los datos obtenidos durante la investigación validan la existencia del modelo compuesto por once factores en el Cuestionario de Esquemas de Young forma larga, segunda edición para Medellín.

El Cuestionario YsQ-L2 está compuesto por 45 ítems que evalúan once factores (esquemas) y es de administración heteroaplicada. La validez tipo alfa del cuestionario fue de 0,91 . El alfa de los factores osciló entre el 0,71 y el 0,85. Los ítems se puntúan según una escala tipo Likert de 6 valores, representando la presencia de los esquemas: $1=$ Completamente falso de mí; 2 = Mayor parte falso de mí; 3 = Ligeramente más verdadero que falso; 4 = Moderadamente verdadero de mí; $5=$ La mayor parte verdadero de mí; $6=$ Me describe perfectamente. Al revisar los 45 ítems de esta prueba no se ha encontrado entre ellos alguno que deba ser cambiado, retirado o anexado, ya que el instrumento puede ser propicio para la población por evaluar, de acuerdo con la edad y el nivel de escolaridad; sin embargo, luego de aplicada la prueba piloto el cuestionario fue modifi- cado en algunos de sus ítems para mejor comprensión de la población adulta.

\section{Plan de análisis}

La información se analizó con el programa de análisis estadístico sPss en su versión 19 y se realizaron análisis de estadísticos descriptivos y comparación de rangos con la prueba U de Mann-Whitney.

\section{Resultados}

Dentro de la base de datos se cuenta con 73 pacientes de grupo HTA. Al sistematizar los datos fueron arrojadas tablas con las medias de cada esquema, la desviación típica, media normativa y la desviación típica normativa, en las que se halló una diferencia significativa de tres esquemas maladaptativos tempranos que sobresalieron de un total de once esquemas (abandono, vulnerabilidad al daño y a la enfermedad e inhibición emocional) (ver tabla 1); esto muestra que las personas con hipertensión poseen en mayor grado estos esquemas. En cuanto a los demás esquemas, no se hallaron diferencias presentes teniendo en cuenta la tabla tomada como referencia, producto de la validación del YSQ en la población colombiana (Castrillón et al., 2005).

En la tabla 2 se comparan los rangos de las puntuaciones de los esquemas entre los grupos de hipertensos y normotensos. Sólo se hallan diferencias significativas en el esquema de vulnerabilidad al daño y a la enfermedad, lo cual indica que este esquema se encuentra presente en mayor medida en el grupo de pacientes hipertensos (ver tablas 2 y 3 ).

Tabla 1. Comparación de medias de EмT en grupo de pacientes hipertensos, con las medias normativas

\begin{tabular}{lccccc}
\hline \multicolumn{1}{c}{ Esquemas } & N & Media & Desviación típ. & Media normativa & $\begin{array}{c}\text { Desviación típica } \\
\text { normativa }\end{array}$ \\
\hline Abandono & 73 & 24,18 & 8,559 & 16,09 & 7,47 \\
\hline Insuficiente autocontrol & 73 & 15,16 & 7,493 & 13,13 & 6,16 \\
\hline Desconfianza/abuso & 73 & 17,92 & 7,019 & 13,00 & 6,00 \\
\hline Privación emocional & 73 & 6,37 & 3,442 & 11,3 & 5,01 \\
\hline $\begin{array}{l}\text { Vulnerabilidad al daño y a la } \\
\text { enfermedad }\end{array}$ & 73 & 20,15 & 5,866 & 10,34 & 5,53 \\
\hline Autosacrificio & 73 & 14,97 & 4,952 & 11,85 & 4,98 \\
\hline Estándares inflexibles I & 73 & 13,86 & 5,078 & 4,76 \\
\hline Estándares inflexibles II & 73 & 13,03 & 4,007 & 4,00 \\
\hline Inhibición emocional & 73 & 10,3 & 4,539 & 9,17 & 3,49 \\
\hline Derecho & 73 & 6,68 & 4,381 & 6,17 & \\
\hline Entrampamiento & 73 & 6,15 & 3,766 & 6,55 & 3,11 \\
\hline
\end{tabular}

Fuente: elaboración propia 
Tabla 2. Comparación de medias en grupo de normotensos e hipertensos

\begin{tabular}{|c|c|c|c|c|}
\hline Esquemas & $\begin{array}{c}\text { Hipertensión } \\
\text { arterial }\end{array}$ & $\mathrm{N}$ & $\begin{array}{c}\text { Rango } \\
\text { promedio }\end{array}$ & Suma de rangos \\
\hline \multirow{2}{*}{ Abandono } & No HTA & 64 & 68,2 & 4365 \\
\hline & HTA & 73 & 69,7 & 5088 \\
\hline \multirow{2}{*}{ Insuficiente autocontrol } & No HTA & 64 & 65,95 & 4221 \\
\hline & HTA & 73 & 71,67 & 5232 \\
\hline \multirow{2}{*}{ Desconfianza/abuso } & No HTA & 64 & 67,44 & 4316 \\
\hline & HTA & 73 & 70,37 & 5137 \\
\hline \multirow{2}{*}{ Privación emocional } & No HTA & 64 & 66,15 & 4233,5 \\
\hline & HTA & 73 & 71,5 & 5219,5 \\
\hline \multirow{2}{*}{ Vulnerabilidad al daño y a la enfermedad } & No HTA & 64 & 60,81 & 3892 \\
\hline & HTA & 73 & 76,18 & 5561 \\
\hline \multirow{2}{*}{ Autosacrificio } & No HTA & 64 & 67,76 & 4336,5 \\
\hline & HTA & 73 & 70,09 & 5116,5 \\
\hline \multirow{2}{*}{ Estándares inflexibles I } & No HTA & 64 & 69,87 & 4471,5 \\
\hline & HTA & 73 & 68,24 & 4981,5 \\
\hline \multirow{2}{*}{ Estándares inflexibles II } & No HTA & 64 & 70,59 & 4518 \\
\hline & HTA & 73 & 67,6 & 4935 \\
\hline \multirow{2}{*}{ Inhibición emocional } & No HTA & 64 & 69,84 & 4469,5 \\
\hline & HTA & 73 & 68,27 & 4983,5 \\
\hline \multirow{2}{*}{ Derecho } & No HTA & 64 & 69,93 & 4475,5 \\
\hline & HTA & 73 & 68,18 & 4977,5 \\
\hline \multirow{2}{*}{ Entrampamiento } & No HTA & 64 & 67,43 & 4315,5 \\
\hline & HTA & 73 & 70,38 & 5137,5 \\
\hline
\end{tabular}

Fuente: elaboración propia

Tabla 3. Prueba U de comparación de rangos

U de Mann-Whitney

\begin{tabular}{|c|c|c|c|}
\hline Abandono & 2285,000 & $-0,221$ & 0,825 \\
\hline Insuficiente autocontrol & 2441,000 & $-0,849$ & 0,396 \\
\hline Desconfianza/abuso & 2236,000 & $-0,432$ & 0,666 \\
\hline Privación emocional & 2135,500 & $-0,805$ & 0,421 \\
\hline Vulnerabilidad al daño y a la enfermedad & 1812,000 & 2,401 & 0,016 \\
\hline Autosacrificio & 2256,500 & $-0,344$ & 0,731 \\
\hline Estándares inflexibles I & 2280,500 & $-0,241$ & 0,810 \\
\hline Estándares inflexibles II & 2234,000 & $-0,446$ & 0,656 \\
\hline Inhibición emocional & 2282,500 & $-0,233$ & 0,816 \\
\hline Derecho & 2276,500 & $-0,265$ & 0,791 \\
\hline Entrampamiento & 2235,500 & $-0,445$ & 0,656 \\
\hline
\end{tabular}

Fuente: elaboración propia

\section{Discusión}

Los elementos más relevantes encontrados en los resultados obtenidos se refieren a los EMT prevalecientes en los pacientes hipertensos, así como a las diferencias halladas en la comparación entre normotensos y pacientes de la patología cardiovascular (HTA).

En cuanto al esquema de abandono, este forma parte de la dimensión de desconexión y rechazo propuesta por Young (Castrillón et al., 2005); este primer esquema que aparece en el grupo de pacientes con HTA hace referencia a la creencia de que los demás no pueden dar un apoyo fiable y estable, las personas que se encuentran alrededor no son consideradas como un apoyo constante, es decir con el que puedan contar todo el tiempo, o bien, si están, se tiene temor frecuente a que les dejen o los abandonen. En nuestra cultura colombiana antioqueña es notorio que se tejen dentro de las familias lazos afectivos fuertes. Incluso algo característico de nuestra población, a diferencia de otros países, es la preocupación constante de una persona por los intereses y las dificultades de los demás en las 
diferentes áreas de la vida del ser humano (salud, empleo, educación o sentimientos). Por lo anterior es pertinente señalar que muchos de los pacientes evaluados son personas que se encuentran rodeadas por sus familias; sin embargo, muchos de ellos han quedado viudos o sus hijos han partido de sus hogares a formar nuevos; en otros casos muchos de ellos tienen hijos que han fallecido a causa de la guerra, y aunque saben que sus familias representan un apoyo para ellos, manifiestan sentimientos de abandono y desprotección, viviendo cada separación como un abandono. La edad de estas personas también ayudaría a entender el fenómeno, puesto que son personas en la tercera edad, y es común encontrar percepciones alrededor de las pérdidas, no sólo de personas sino de la funcionalidad de su propio cuerpo; esto podría estar relacionado con los altos índices de suicidio en la vejez. Es importante entender también que lo más probable es que la presencia de este esquema nos hable de una relación estrecha con la disfuncionalidad familiar (Ruiz y Orozco, 2009).

El esquema de vulnerabilidad al daño o a la enfermedad forma parte de la dimensión perjuicio en autonomía y desempeño. Se refiere a que los individuos esperen tener experiencias negativas que no controlan, tales como crisis médicas, emocionales o naturales. Estudios como los realizados en 1959 por Friedman y Rosenman se refirieron al entorno percibido como amenazante por parte de los propensos coronarios (Colunga, 2007), situaciones que se les salen de control, por ejemplo ser atacados o perder todo el dinero y quedarse en la ruina. Como lo mencionan los autores que se han referido al tema, para estas personas el entorno es amenazante; se ven a sí mismas como vulnerables y que fácilmente podrían ser alcanzados por alguna crisis; en el territorio donde viven se conoce cada día el fallecimiento de personas allegadas a causa de la violencia, y asimismo se rumora de posibles desastres en el terreno por falta de firmeza a causa de la explotación minera, como también de posibles daños corporales a causa de la exposición al mercurio en el aire (elemento utilizado en las labores mineras), que causa posibles daños en piel, cerebro, respiración, ojos, memoria, concentración, entre otros. Además hay quienes en condiciones de pobreza tienen sus viviendas ubicadas en lugares de alto riesgo. Estas y otras condiciones podrían ayudar para que estas personas sientan una constante amenaza que pone en peligro sus vidas.

El esquema de inhibición emocional forma parte de la dimensión de sobrevigilancia e inhibición; se encuentra en individuos que evitan la espontaneidad para mantener la sensación de que prevén y controlan sus vidas, o para evitar la vergüenza. Son personas que viven aisladas, no suelen solicitar apoyo y sus redes sociales son limitadas, inhibiendo sus sentimientos, manteniendo la sensación de que tienen todo bajo control y que no se afectan fácilmente. "Tienen una alta actividad fisiológica ante situaciones específicas, originada por la hostilidad y viven conflictos psicológicos" (Colunga, 2007). Se han encontrado otros estudios que confirman la relación entre la hipertensión y la inhibición emocional, no en los mismos términos pero sí hablando de la defensividad emocional (Mann y James, 1998; Tapia y Labiano, 2004). Esto incluso podría entenderse como factor predisponente a la hipertensión, puesto que aumentaría los niveles de presión sanguínea (Jamner, Shapiro, Goldstein y Hug, 1991).

En cuanto a la comparación de los grupos participantes del estudio (hipertensos y normotensos) se hallan diferencias significativas sólo en el esquema de vulnerabilidad al daño y a la enfermedad. Dicho esquema hace referencia a la anticipación de catástrofes inminentes e incontrolables. Las anticipaciones son de orden médico, emocionales y catástrofes externas (Castrillón et al., 2005), lo que indica que quienes presentan puntuaciones altas en dicho esquema son personas con un alto índice de ansiedad, ya que se anticipan a la gravedad de las situaciones; con frecuencia hay presencia de pensamientos y sentimientos de preocupación, miedo e inseguridad, es decir, aparece una serie de "rumiaciones" que llevan al individuo hipertenso a un estado de alerta, desasosiego o tensión con mayor frecuencia que al sujeto de la población normal (Casado Morales, 1994). Esto podría estar ocasionado por el personal de salud cuando los pacientes asisten a los controles médicos, y reciben advertencias del riesgo para su salud en caso de no seguir con las recomendaciones. Es decir, la adherencia a los tratamientos contra la hipertensión influiría en el desarrollo o la activación de este esquema. Esta hipótesis generaría una dificultad frente a las recomendaciones del personal de salud, puesto que se asume que una mayor adherencia aumenta un pronóstico favorable de la afección; no obstante, es probable que las recomendaciones del personal de salud estén orientadas por el uso masivo de tecnicismos y una actitud alarmante hacia los pacientes. Se recomienda precisamente evitar dicha actitud (Zambrano, Duitama, Posada y Flórez, 2012).

La vulnerabilidad al daño y a la enfermedad es de principal atención en este artículo puesto que habla- 
ría exclusivamente de las personas con diagnóstico de hipertensión y no sólo de personas en la tercera edad como las anteriores, y aunque aparecen otros esquemas significativos, podría pensarse que son secundarios en la configuración del sistema de creencias de las personas con hipertensión.

En este grupo de personas operaría un modelo de esquemas caracterizado por la vulnerabilidad al daño y a la enfermedad, y que este se mantendría por la presencia de los esquemas de abandono e inhibición emocional. La figura 1 ilustra este modelo.

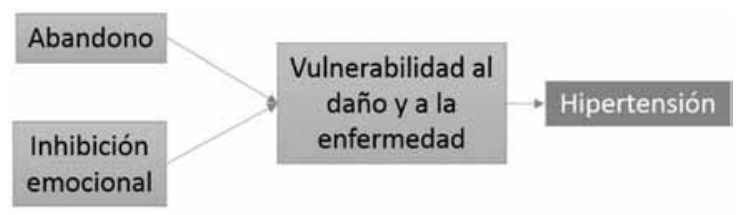

Figura 1. Propuesta de modelo de EMT asociados a la hipertensión esencial

Fuente: elaboración propia

Diferentes autores han investigado la relación entre la ansiedad y la presencia de HTA u otras alteraciones cardiovasculares. Lacey y Lacey (1958) estudiaron respuestas autonómicas en sujetos, los cuales exhibían un patrón de respuesta idiosincrásico en todas las tareas: algunos sujetos respondían principalmente con elevación de tasa cardiaca, otros con elevación de conductancia o de presión arterial, lo cual justifica que ante algunas experiencias interpretadas como dañinas, peligrosas o fuera de control, a las personas se les incremente la tasa cardiaca.

Otros estudios realizados (Baer, Collin, Bourianoff y Ketchel, 1979) apuntan a que los sujetos hipertensos obtienen puntuaciones más altas que los normotensos en activación de ira, ansiedad y resentimiento. Por otro lado, Schalling y Svensson (1984, citados en Casado Morales, 1994) encontraron diferencias significativas entre hipertensos y normotensos en escalas de ansiedad, tensión, neuroticismo e "inhibición de agresión". Los hipertensos puntuaban más alto en estas escalas que los normotensos. Por tanto, los autores describen un perfil del hipertenso como un individuo ansioso, que reprime o inhibe la ira y que no es asertivo.

Otros autores retomados por Casado Morales (1994), como Thailer et al. (1985), sostienen que los pacientes hipertensos con elevación de la renina plasmática presentan mayores niveles de ira contenida, susceptibilidad, paranoidismo, depresión y ansiedad. Miguel-Tobal et al. (1994) describen el perfil del sujeto hipertenso caracterizado por un alto rasgo general de ansiedad, alta reactividad en los sistemas de respuesta cognitivo y fisiológico, $y$, en menor medida, en el sistema motor; igualmente por reacciones de ansiedad intensas ante las situaciones de prueba o evaluación, situaciones potencialmente fóbicas, las habituales en su vida diaria y, en menor medida, ante las situaciones interpersonales. De hecho el grupo de hipertensos de aquella investigación presentaron mayores puntuaciones que el grupo normal en rasgo general de ansiedad, es decir, en la propensión a responder de forma ansiosa ante las distintas situaciones.

De la misma manera, varios estudios citados por Fernandez Abascal, Martin Díaz y Domínguez Sánchez (2003) han mencionado la implicación de la ansiedad y la enfermedad cardiovascular. Una importante evidencia epidemiológica recogida a lo largo de 32 años en el estudio de seguimiento Normative Aging Study, el cual revela que la preocupación y la ansiedad están asociadas con enfermedad coronaria fatal y muerte cardiaca súbita (Kawachi, Sparrow, Vokonas y Weiss, 1994; Kubzansky, Kawachi, Spiro, Weis, Vokonas y Sparrow, 1997, citados por Fernández Abascal et al., 2003), al igual que la ansiedad, podrían estar implicadas en el desencadenamiento de un suceso coronario agudo. Finalmente se encuentra evidencia que asocia la ansiedad con un incremento del riesgo de infarto de miocardio (Mittleman, Maclure, Sherwood, Mulry, Tofler et al., 1995, citados por Fernández Abascal, 2003).

\section{Conclusiones}

Dado que el ser humano pasa a lo largo de la vida por diferente grupos, se podría trabajar en pro de incentivar y educar sobre el empleo de estrategias de afrontamiento útiles, como las enfocadas a resolver el problema, las de referencia a otros, y el afrontamiento no productivo (Solís Manrique y Vidal Miranda, 2006).Dentro de estas están: invertir en amigos íntimos, para así ampliar cada vez más las redes de apoyo, ya que esto implica ampliarlas a nivel psicológico también; fijarse en lo positivo, es decir, tener una visión optimista del presente y de la tendencia del futuro; buscar diversiones relajantes, actividades de ocio que permitan la distensión, la distracción física; realizar actividades que permitan oxigenar el cuerpo y mantenerse en forma; buscar apoyo social, relacionándose con 
más personas, y compartir dificultades puesto que de esta manera podrían encontrarse más vías de solución; buscar apoyo profesional, acción social; buscar apoyo espiritual. De esta manera se podría reducir o evitar que se desarrollen altos niveles de ansiedad, ya que esta se encuentra directamente relacionada con el esquema de vulnerabilidad al daño y a la enfermedad. Por tanto, si las personas tienen dentro de sus posibilidades muchas vías a la hora de solucionar un problema, su nivel de ansiedad podría ser menor, al igual que si percibe mayores redes de apoyo y mayor inversión en la relajación y la dispersión (como practicar actividades de grupo, gimnasio, de participación social comunitaria, de descanso, respiración, teniendo en cuenta que deben ser actividades placenteras para las personas que las desarrollan) invirtiendo así menor tiempo para la concentración del problema y de preocupaciones que no le corresponden pero que le desestabilizan.

\section{Agradecimientos}

A todas las personas que contribuyeron con el desarrollo del estudio. A cada uno de los participantes de la muestra y a las personas que dirigen los grupos en los cuales fue aplicado el instrumento. Sin ellos no hubiese sido posible llevar a cabo este estudio.

\section{Referencias}

Aristizábal, D., García, E., McEwen, J., Caulfield, M., Méndez, J., Medina, E., Zapata, N. y Correa, M. (2006). Bases genéticas de la hipertensión arterial esencial en Colombia: avances en nueve años de estudio. Revista Colombiana de Cardiología, 12(6), 409-430.

Baer, P. E., Collins, F. H. Bourenoff, G. C. y Ketchel, M. F. (1979). Asessing personality factors in essential hypertension with a brief self-report instrument. Psychosomatic Medicine, 16, 721-730.

Casado Morales, M. I. (1994). Ansiedad, stress y trastornos psicofisiológicos. [Tesis doctoral]. Universidad Complutense de Madrid, España. Recuperado de http://biblioteca.ucm.es/tesis/19911996/S/4/S4005801.pdf
Castrillón, D. A., Chaves, L., Ferrer, A., Londoño, N. H., Maestre, K., Marín, C. y Schnitter, M. (2005). Validación del Young questionnaire long form-second edition (YSQ-L2) en población colombiana. Revista Latinoamericana de Psicología, 37(3), 541-560.

Colunga, C. (2007). Algunos factores psicosociales en la hipertensión. En Hipertensión Arterial en la Clínica (pp. 2-9). Guadalajara, México: McGraw-Hill.

Fernández Abascal, E. G., Martín Díaz, M. D. y Domínguez Sánchez, F. J. (2003). Factores de riesgo e intervenciones psicológicas eficaces en los trastornos cardiovasculares. Psicothema, 15(4), 615-630.

Hernández Sampieri, R., Fernández Collao, C. y Baptista Lucio, P. (2003). Metodología de la investigación. México D. F.: McGraw-Hill Interamericana.

Jamner, L. D., Shapiro, D., Goldstein, I. B. y Hug, R. (1991). Ambulatory blood pressure and heart rate in paramedics: Effects of cynical hostility and defensiveness. Psychosomatic Medicine, 53, 393-406.

Lacey, J. y Lacey, B. (1958). Verification and extension of de principie of autonornic response-stereotipy. American Journal of Psychology, 71, 50-73.

Mann, S. J. y James, G. D. (1998). Defensiveness and essential hypertension. Journal of Psychosomatic Research, 45(2), 139-148.

Miguel-Tobal, J. J., Cano-Vindel, A., Casado Morales, M. I. y Escalona Martínez, A. (1994). Emociones e hipertensión. Implantación de un programa cognitivo-conductual en pacientes hipertensos. Anales de Psicología, 10(2), 199-216.

Ruiz, F. y Orozco, H. (2009). El abandono del adulto mayor como manifestación de violencia intrafamiliar. Archivos en Medicina Familiar, 11(4), 147-149.

Solís Manrique, C. y Vidal Miranda, A. (2006). Estilos y estrategias de afrontamiento en adolescentes. Revista de Psiquiatria y Salud Mental. Hermilio Valdizan, 7(1), 33-39.

Tapia, M. L. y Labiano, L. M. (2004). Factores emocionales e hipertensión esencial. Terapia Psicológica, 22(2), 103109.

Zambrano, R., Duitama, J., Posada, J. y Flórez, J. (2012). Percepción de la adherencia a tratamientos en pacientes con factores de riesgo cardiovascular. Revista de la Facultad Nacional de Salud Pública, 30(2), 163-174. 